Supporting Information for

\title{
Molecular Vertical Excitation Energies Studied with First Order RASSCF (RAS[1,1]): Balancing Covalent and lonic Excited States
}

Thierry Tran, Javier Segarra-Martí, Michael J. Bearpark and Michael A. Robb*

Department of Chemistry, Molecular Sciences Research Hub, Imperial College

London, White City Campus, 80 Wood Lane, London W12 0BZ, United Kingdom

\section{Content}

1. Vertical excitation energies and molecular orbitals for $n \pi^{*}$ states

2. Error comparison

3. References 


\section{Vertical excitation energies and molecular orbitals for $n \pi^{*}$ states}

Table S1: Vertical excitation energies (eV) for the lowest singlet valence states of ethylene obtained at the ground state MP2/6-31G* equilibrium geometry. The symmetry point group is $D_{2 h}$.

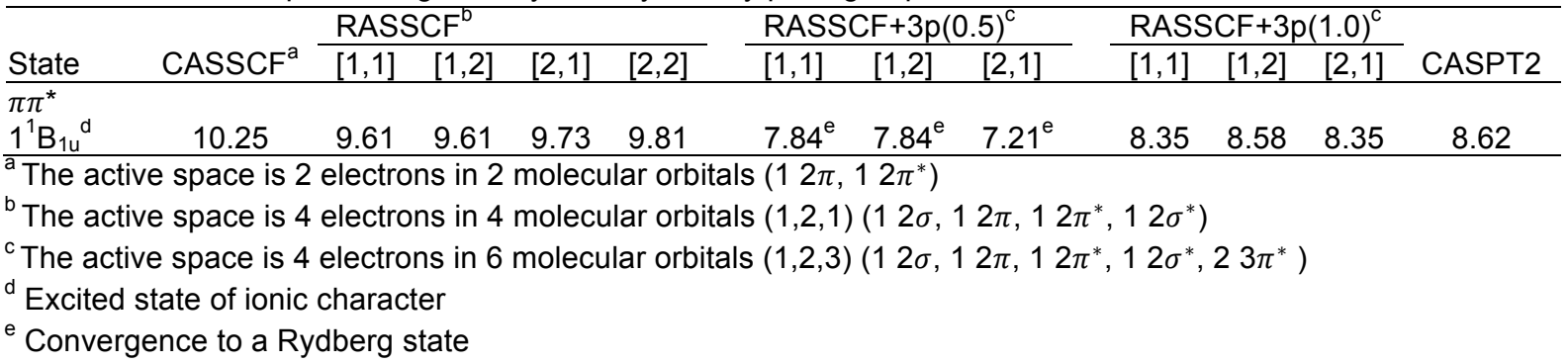

Table S2: Vertical excitation energies $(\mathrm{eV})$ for the lowest singlet valence states of E-butadiene obtained at the ground state MP2/6-31G* equilibrium geometry. The symmetry point group is $\mathrm{C}_{2 \mathrm{~h}}$.

\begin{tabular}{|c|c|c|c|c|c|c|c|c|c|c|c|c|}
\hline \multirow[b]{2}{*}{ State } & \multirow[b]{2}{*}{ CASSCF $^{a}$} & \multicolumn{4}{|c|}{ RASSCF $^{\mathrm{b}}$} & \multicolumn{3}{|c|}{ RASSCF $+3 p(0.5)^{C}$} & \multicolumn{3}{|c|}{ RASSCF $+3 p(1.0)^{C}$} & \multirow[b]{2}{*}{ CASPT2 } \\
\hline & & {$[1,1]$} & {$[1,2]$} & {$[2,1]$} & {$[2,2]$} & {$[1,1]$} & {$[1,2]$} & {$[2,1]$} & {$[1,1]$} & {$[1,2]$} & {$[2,1]$} & \\
\hline $\begin{array}{l}\pi \pi^{*} \\
1^{1} \mathrm{~B}_{\mathrm{u}}{ }^{\mathrm{d}}\end{array}$ & 8.63 & 7.93 & 7.96 & 7.94 & 8.09 & 6.82 & 6.68 & 6.83 & 7.17 & 7.21 & 7.15 & 6.47 \\
\hline $2^{1} \mathrm{~A}_{\mathrm{g}}^{\mathrm{e}}$ & 6.69 & 7.03 & 7.04 & 7.03 & 7.02 & 6.75 & 6.77 & 6.92 & 6.72 & 6.80 & 6.70 & 6.83 \\
\hline
\end{tabular}

${ }^{\mathrm{a}}$ The active space is 4 electrons in 4 molecular orbitals $\left(22 \pi, 22 \pi^{*}\right)$

${ }^{\mathrm{b}}$ The active space is 10 electrons in 10 molecular orbitals $(3,4,3)\left(32 \sigma, 22 \pi, 22 \pi^{*}, 32 \sigma^{*}\right)$

${ }^{\mathrm{c}}$ The active space is 10 electrons in 14 molecular orbitals $(3,4,7)\left(32 \sigma, 22 \pi, 22 \pi^{*}, 32 \sigma^{*}, 43 \pi^{*}\right)$

${ }^{d}$ Excited state of ionic character

${ }^{\mathrm{e}}$ Excited state of covalent character

Table S3: Vertical excitation energies $(\mathrm{eV})$ for the lowest singlet valence states of All-E-hexatriene obtained at the ground state MP2/6-31G* equilibrium geometry. The symmetry point group is $\mathrm{C}_{2 \mathrm{~h}}$.

\begin{tabular}{|c|c|c|c|c|c|c|c|c|c|c|c|c|}
\hline \multirow[b]{2}{*}{ State } & \multirow[b]{2}{*}{ CASSCF $^{a}$} & \multicolumn{4}{|c|}{ RASSCF $^{\text {D }}$} & \multicolumn{3}{|c|}{ RASSCF $+3 p(0.5)^{C}$} & \multicolumn{3}{|c|}{ RASSCF+3p(1.0) ${ }^{C}$} & \multirow[b]{2}{*}{ CASPT2 } \\
\hline & & {$[1,1]$} & {$[1,2]$} & {$[2,1]$} & {$[2,2]$} & {$[1,1]$} & {$[1,2]$} & {$[2,1]$} & {$[1,1]$} & {$[1,2]$} & {$[2,1]$} & \\
\hline$\pi \pi^{*}$ & & & & & & & & & & & & \\
\hline $1^{1} B_{u^{d}}$ & 7.58 & 6.57 & 6.89 & 6.87 & 6.97 & 6.20 & 6.21 & 6.17 & 6.27 & 6.30 & 6.27 & 5.31 \\
\hline $2^{1} A_{g}^{e}$ & 5.50 & 5.80 & 5.80 & 5.80 & 5.80 & 5.76 & 5.67 & 5.76 & 5.76 & 5.77 & 5.77 & 5.42 \\
\hline
\end{tabular}

${ }^{\mathrm{a}}$ The active space is 6 electrons in 6 molecular orbitals $\left(32 \pi, 32 \pi^{*}\right)$

${ }^{\mathrm{b}}$ The active space is 16 electrons in 16 molecular orbitals $(5,6,5)\left(52 \sigma, 32 \pi, 32 \pi^{*}, 52 \sigma^{*}\right)$

${ }^{\mathrm{c}}$ The active space is 16 electrons in 22 molecular orbitals $(5,6,11)\left(52 \sigma, 32 \pi, 32 \pi^{*}, 52 \sigma^{*}, 63 \pi^{*}\right)$

${ }^{d}$ Excited state of ionic character

${ }^{\mathrm{e}}$ Excited state of covalent character

Table S4: Vertical excitation energies $(\mathrm{eV})$ for the lowest singlet valence states of All-E-octotetraene obtained at the ground state MP2/6-31G* equilibrium geometry. The symmetry point group is $\mathrm{C}_{2 \mathrm{~h}}$.

\begin{tabular}{|c|c|c|c|c|c|c|c|c|c|c|c|c|}
\hline \multirow[b]{2}{*}{ State } & \multirow[b]{2}{*}{ CASSCF $^{a}$} & \multicolumn{4}{|c|}{ RASSCF $^{\mathrm{b}}$} & \multicolumn{3}{|c|}{ RASSCF $+3 p(0.5)^{c}$} & \multicolumn{3}{|c|}{ RASSCF $+3 p(1.0)^{c}$} & \multirow[b]{2}{*}{ CASPT2 } \\
\hline & & {$[1,1]$} & {$[1,2]$} & {$[2,1]$} & {$[2,2]$} & {$[1,1]$} & {$[1,2]$} & {$[2,1]$} & {$[1,1]$} & {$[1,2]$} & {$[2,1]$} & \\
\hline $\begin{array}{l}\pi \pi^{*} \\
2^{1} \mathrm{~A}_{\mathrm{g}}{ }^{\mathrm{d}} \\
1^{1} \mathrm{~B}_{\mathrm{u}}\end{array}$ & $\begin{array}{l}4.74 \\
5.92\end{array}$ & $\begin{array}{l}4.97 \\
6.19\end{array}$ & $\begin{array}{l}4.97 \\
6.21\end{array}$ & $\begin{array}{l}4.98 \\
6.18\end{array}$ & $\begin{array}{l}4.92 \\
6.23\end{array}$ & $\begin{array}{l}4.86 \\
5.60\end{array}$ & $\begin{array}{l}4.85 \\
5.64\end{array}$ & $\begin{array}{l}4.94 \\
5.61\end{array}$ & $\begin{array}{l}4.93 \\
5.63\end{array}$ & $\begin{array}{l}4.87 \\
5.68\end{array}$ & $\begin{array}{l}4.95 \\
5.65\end{array}$ & $\begin{array}{l}4.64 \\
4.70\end{array}$ \\
\hline
\end{tabular}

${ }^{a}$ The active space is 8 electrons in 8 molecular orbitals $\left(42 \pi, 42 \pi^{*}\right)$

${ }^{\mathrm{b}}$ The active space is 22 electrons in 22 molecular orbitals $(7,8,7)\left(72 \sigma, 42 \pi, 42 \pi^{*}, 72 \sigma^{*}\right)$

${ }^{c}$ The active space is 22 electrons in 30 molecular orbitals $(7,8,15)\left(72 \sigma, 42 \pi, 42 \pi^{*}, 72 \sigma^{*}, 83 \pi^{*}\right)$

${ }^{\mathrm{d}}$ Excited state of covalent character

e Excited state of ionic character 
Table S5: Vertical excitation energies $(\mathrm{eV})$ for the lowest singlet valence states of cyclopropene obtained at the ground state MP2/6-31G* equilibrium geometry. The symmetry point group is $\mathrm{C}_{2 \mathrm{v}}$.

\begin{tabular}{|c|c|c|c|c|c|c|c|c|c|c|c|}
\hline \multirow[b]{2}{*}{ State } & \multirow[b]{2}{*}{ CASSCF $^{a}$} & \multicolumn{3}{|l|}{ RASSCF $^{\text {D }}$} & \multicolumn{3}{|c|}{ RASSCF $+3 p(0.5)^{\mathrm{C}}$} & \multicolumn{3}{|c|}{ RASSCF+3p(1.0) ${ }^{\mathrm{C}}$} & \multirow[b]{2}{*}{ CASPT2 } \\
\hline & & {$[1,1] \quad[1,2]$} & {$[2,1]$} & {$[2,2]$} & {$[1,1]$} & {$[1,2]$} & {$[2,1]$} & {$[1,1]$} & {$[1,2]$} & {$[2,1]$} & \\
\hline $\begin{array}{l}\pi \pi^{*} \\
1^{1} \mathrm{~B}_{2}{ }^{\mathrm{d}}\end{array}$ & 9.20 & 8.16 & 8.25 & 8.62 & 7.16 & 7.47 & 7.15 & 7.45 & 7.65 & 7.45 & 7.06 \\
\hline \multicolumn{12}{|c|}{${ }^{a}$ The active space is 2 electrons in 2 molecular orbitals $\left(12 \pi, 12 \pi^{*}\right)$} \\
\hline \multicolumn{12}{|c|}{${ }^{\mathrm{b}}$ The active space is 8 electrons in 8 molecular orbitals $(3,2,3)\left(32 \sigma, 12 \pi, 12 \pi^{*}, 32 \sigma^{*}\right)$} \\
\hline \multicolumn{12}{|c|}{${ }^{\mathrm{c}}$ The active space is 8 electrons in 10 molecular orbitals $(3,2,5)\left(32 \sigma, 12 \pi, 12 \pi^{*}, 32 \sigma^{*}, 23 \pi^{*}\right)$} \\
\hline
\end{tabular}

Table S6: Vertical excitation energies $(\mathrm{eV})$ for the lowest singlet valence states of cyclopentadiene obtained at the ground state MP2/6-31G* equilibrium geometry. The symmetry point group is $C_{2 v}$.

\begin{tabular}{|c|c|c|c|c|c|c|c|c|c|c|c|c|}
\hline \multirow[b]{2}{*}{ State } & \multirow[b]{2}{*}{ CASSCF $^{a}$} & \multicolumn{4}{|c|}{ RASSCF $^{\text {b }}$} & \multicolumn{3}{|c|}{ RASSCF $+3 p(0.5)^{c}$} & \multicolumn{3}{|c|}{ RASSCF $+3 p(1.0)^{c}$} & \multirow[b]{2}{*}{ CASPT2 } \\
\hline & & {$[1,1]$} & {$[1,2]$} & {$[2,1]$} & {$[2,2]$} & {$[1,1]$} & {$[1,2]$} & {$[2,1]$} & {$[1,1]$} & {$[1,2]$} & {$[2,1]$} & \\
\hline$\pi \pi^{*}$ & & & & & & & & & & & & \\
\hline $2^{1} \mathrm{~A}_{1}{ }^{\mathrm{e}}$ & $\begin{array}{r}1.14 \\
6.61 \\
\end{array}$ & $\begin{array}{l}0.09 \\
6.97\end{array}$ & $\begin{array}{l}0.15 \\
6.96\end{array}$ & $\begin{array}{l}0.10 \\
6.96 \\
\end{array}$ & $\begin{array}{l}0.90 \\
6.94 \\
\end{array}$ & $\begin{array}{l}0.13 \\
6.67 \\
\end{array}$ & $\begin{array}{l}0 .<5 \\
6.73 \\
\end{array}$ & $\begin{array}{l}0.12 \\
6.64 \\
\end{array}$ & $\begin{array}{l}0.18 \\
6.69 \\
\end{array}$ & $\begin{array}{l}0.25 \\
6.75 \\
\end{array}$ & $\begin{array}{l}0.11 \\
6.69 \\
\end{array}$ & $\begin{array}{l}0.01 \\
6.31 \\
\end{array}$ \\
\hline
\end{tabular}

${ }^{a}$ The active space is 4 electrons in 4 molecular orbitals $\left(22 \pi, 22 \pi^{*}\right)$

${ }^{\mathrm{b}}$ The active space is 14 electrons in 14 molecular orbitals $(5,4,5)\left(52 \sigma, 22 \pi, 22 \pi^{*}, 52 \sigma^{*}\right)$

${ }^{\mathrm{c}}$ The active space is 14 electrons in 18 molecular orbitals $(5,4,9)\left(52 \sigma, 22 \pi, 22 \pi^{*}, 52 \sigma^{*}, 43 \pi^{*}\right)$

${ }^{\mathrm{d}}$ Excited state of ionic character

${ }^{\mathrm{e}}$ Excited state of covalent character

Table S7: Vertical excitation energies $(\mathrm{eV})$ for the lowest singlet valence states of benzene obtained at the ground state MP2/6-31G* equilibrium geometry. The symmetry point group is $D_{6 h}$.

\begin{tabular}{|c|c|c|c|c|c|c|c|c|c|c|c|c|}
\hline \multirow[b]{2}{*}{ State } & \multirow[b]{2}{*}{ CASSCF $^{a}$} & \multicolumn{4}{|c|}{ RASSCF $^{\text {b }}$} & \multicolumn{3}{|c|}{ RASSCF $+3 p(0.5)^{c}$} & \multicolumn{3}{|c|}{ RASSCF $+3 p(1.0)^{c}$} & \multirow[b]{2}{*}{ CASPT2 } \\
\hline & & {$[1,1]$} & {$[1,2]$} & {$[2,1]$} & {$[2,2]$} & {$[1,1]$} & {$[1,2]$} & {$[2,1]$} & {$[1,1]$} & {$[1,2]$} & {$[2,1]$} & \\
\hline$\pi \pi^{*}$ & 48 & 534 & 533 & 533 & 529 & $\underbrace{\dagger}$ & $ـ^{\dagger}$ & $-^{\dagger}$ & 518 & 514 & 515 & 505 \\
\hline $1^{1} \mathrm{~B}_{1 \mathrm{u}}^{\mathrm{e}}$ & 8.27 & $\begin{array}{l}0.04 \\
7.74\end{array}$ & $\begin{array}{l}0.00 \\
7.75\end{array}$ & $\begin{array}{l}0.00 \\
7.73\end{array}$ & 7.81 & $-f$ & $-f$ & $-f$ & $\begin{array}{l}0.18 \\
7.19\end{array}$ & $\begin{array}{l}0.14 \\
7.18\end{array}$ & $\begin{array}{l}5.15 \\
7.16\end{array}$ & $\begin{array}{l}0.05 \\
6.45\end{array}$ \\
\hline $1^{1} E_{2 g}$ & 8.10 & & & & & & & & & & & 8.21 \\
\hline
\end{tabular}

${ }^{\mathrm{a}}$ The active space is 6 electrons in 6 molecular orbitals $\left(32 \pi, 32 \pi^{*}\right)$. State-average over 5 states

${ }^{\mathrm{b}}$ The active space is 18 electrons in 18 molecular orbitals $(6,6,6)\left(62 \sigma, 32 \pi, 32 \pi^{*}, 62 \sigma^{*}\right)$

${ }^{\mathrm{c}}$ The active space is 18 electrons in 24 molecular orbitals $(6,6,12)\left(62 \sigma, 32 \pi, 32 \pi^{*}, 62 \sigma^{*}, 63 \pi^{*}\right)$

${ }^{\mathrm{d}}$ Excited state of covalent character

${ }^{\mathrm{e}}$ Excited state of ionic character

${ }^{f}$ Not computed due to linear dependency of basis set

Table S8: Vertical excitation energies $(\mathrm{eV})$ for the lowest singlet valence states of furan obtained at the ground state MP2/6-31G* equilibrium geometry. The symmetry point group is $\mathrm{C}_{2 \mathrm{v}}$.

\begin{tabular}{|c|c|c|c|c|c|c|c|c|c|c|c|c|}
\hline \multirow[b]{2}{*}{ State } & \multirow[b]{2}{*}{ CASSCF $^{a}$} & \multicolumn{4}{|c|}{ RASSCF $^{\text {D }}$} & \multicolumn{3}{|c|}{ RASSCF $+3 p(0.5)^{c}$} & \multicolumn{3}{|c|}{ RASSCF+3p(1.0) } & \multirow[b]{2}{*}{ CASPT2 } \\
\hline & & {$[1,1]$} & {$[1,2]$} & {$[2,1]$} & {$[2,2]$} & {$[1,1]$} & {$[1,2]$} & {$[2,1]$} & {$[1,1]$} & {$[1,2]$} & {$[2,1]$} & \\
\hline$\pi \pi^{*}$ & & & & & & & & & & & & \\
\hline $1^{1} \mathrm{~B}_{2}^{d}$ & 8.36 & 7.64 & 7.64 & 7.63 & 7.82 & 6.51 & 6.80 & 6.46 & 6.98 & 7.09 & 6.97 & 6.43 \\
\hline $2^{1} \mathrm{~A}_{1}{ }^{\mathrm{e}}$ & 6.81 & 7.19 & 7.19 & 7.19 & 7.16 & 6.77 & 6.99 & 6.74 & 6.81 & 6.92 & 6.73 & 6.52 \\
\hline
\end{tabular}

${ }^{\mathrm{a}}$ The active space is 6 electrons in 5 molecular orbitals $\left(32 \pi, 22 \pi^{*}\right)$

${ }^{\mathrm{b}}$ The active space is 16 electrons in 15 molecular orbitals $(5,5,5)\left(52 \sigma, 32 \pi, 22 \pi^{*}, 52 \sigma^{*}\right)$

${ }^{\mathrm{c}}$ The active space is 16 electrons in 20 molecular orbitals $(5,5,10)\left(52 \sigma, 32 \pi, 22 \pi^{*}, 52 \sigma^{*}, 53 \pi^{*}\right)$

${ }^{\mathrm{d}}$ Excited state of ionic character

${ }^{\mathrm{e}}$ Excited state of covalent character 
Table S9: Vertical excitation energies $(\mathrm{eV})$ for the lowest singlet valence states of pyrrole obtained at the ground state MP2/6-31G* equilibrium geometry. The symmetry point group is $C_{2 v}$.

\begin{tabular}{|c|c|c|c|c|c|c|c|c|c|c|c|c|}
\hline \multirow[b]{2}{*}{ State } & \multirow[b]{2}{*}{ CASSCF $^{a}$} & \multicolumn{4}{|c|}{ RASSCF $^{D}$} & \multicolumn{3}{|c|}{ RASSCF+3p $(0.5)^{C}$} & \multicolumn{3}{|c|}{ RASSCF+3p(1.0) } & \multirow[b]{2}{*}{ CASPT2 } \\
\hline & & {$[1,1]$} & {$[1,2]$} & {$[2,1]$} & {$[2,2]$} & {$[1,1]$} & {$[1,2]$} & {$[2,1]$} & {$[1,1]$} & {$[1,2]$} & {$[2,1]$} & \\
\hline$\pi \pi^{*}$ & 663 & 700 & 700 & 700 & 607 & $\dagger$ & $\oplus^{\dagger}$ & ${ }^{\dagger}$ & 661 & 676 & 660 & 631 \\
\hline $1^{1} \mathrm{~B}_{2}{ }^{\mathrm{e}}$ & 8.17 & 7.72 & 7.73 & 7.71 & 7.84 & $-{ }^{f}$ & $-f$ & $-f$ & 6.96 & 7.09 & 6.92 & 6.33 \\
\hline
\end{tabular}

${ }^{\mathrm{a}}$ The active space is 6 electrons in 5 molecular orbitals $\left(32 \pi, 22 \pi^{*}\right)$

${ }^{\mathrm{b}}$ The active space is 16 electrons in 15 molecular orbitals $(5,5,5)\left(52 \sigma, 32 \pi, 22 \pi^{*}, 52 \sigma^{*}\right)$

${ }^{\mathrm{c}}$ The active space is 16 electrons in 20 molecular orbitals $(5,5,10)\left(52 \sigma, 32 \pi, 22 \pi^{*}, 52 \sigma^{*}, 53 \pi^{*}\right)$

${ }^{d}$ Excited state of covalent character

${ }^{\mathrm{e}}$ Excited state of ionic character

${ }^{f}$ Convergence to a Rydberg state 
Table S10: Vertical excitation energies $(\mathrm{eV})$ for the lowest singlet valence states of imidazole obtained at the ground state MP2/6-31G* equilibrium geometry. The symmetry point group is $C_{s}$.

\begin{tabular}{|c|c|c|c|c|c|c|c|c|c|c|}
\hline \multirow{2}{*}{$\frac{\text { State }}{\pi \pi^{*}}$} & \multirow[b]{2}{*}{ CASSCF $^{a}$} & \multicolumn{2}{|l|}{ RASSCF $^{D}$} & \multicolumn{3}{|c|}{ RASSCF $+3 p(0.5)^{c}$} & \multicolumn{3}{|c|}{$\operatorname{RASSCF}+3 p(1.0)^{c}$} & \multirow[b]{2}{*}{ CASPT2 } \\
\hline & & {$[1,1] \quad[1,2]$} & {$[2,1]$} & {$[1,1]$} & {$[1,2]$} & {$[2,1]$} & {$[1,1]$} & {$[1,2]$} & {$[2,1]$} & \\
\hline $\begin{array}{l}\pi \pi^{*} \\
2^{1} A^{, d}\end{array}$ & 6.92 & 7.30 & 7.27 & 6.63 & 6.68 & 6.83 & 7.00 & 6.81 & 7.00 & 6.19 \\
\hline $1^{7} A^{\prime \prime}$ & 7.18 & 7.68 & 7.17 & 6.91 & 7.16 & 6.82 & 7.03 & 7.09 & 6.90 & 6.81 \\
\hline
\end{tabular}

${ }^{a}$ The active space is 8 electrons in 6 molecular orbitals $\left(12 n, 32 \pi, 22 \pi^{*}\right)$. State-average over 4 states

${ }^{\mathrm{b}}$ The active space is 18 electrons in 16 molecular orbitals $(5,6,5)\left(52 \sigma, 12 n, 32 \pi, 22 \pi^{*}, 52 \sigma^{*}\right)$

${ }^{\mathrm{c}}$ The active space is 18 electrons in 21 molecular orbitals $(5,6,10)\left(52 \sigma, 12 n, 32 \pi, 22 \pi^{*}, 52 \sigma^{*}, 53 \pi^{*}\right)$

${ }^{\mathrm{d}}$ Excited state of covalent character (local $n \pi^{*}$ transition)
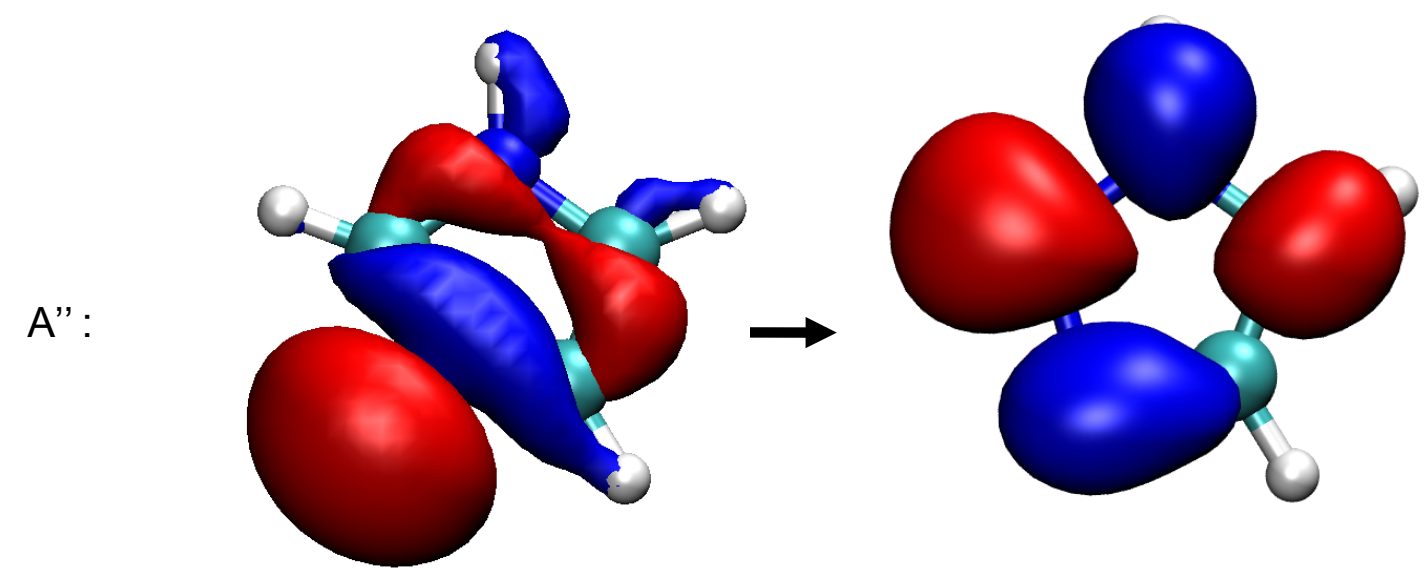

Figure S1: Molecular orbitals (isovalue of 0.03 ) involved in the two lowest $n \pi^{*}$ transition (A" character) of imidazole. 
Table S11: Vertical excitation energies $(\mathrm{eV})$ for the lowest singlet valence states of pyridine obtained at the ground state MP2/6-31G* equilibrium geometry. The symmetry point group is $\mathrm{C}_{2 \mathrm{v}}$.

\begin{tabular}{|c|c|c|c|c|c|c|c|c|c|c|c|}
\hline \multirow{2}{*}{$\frac{\text { State }}{\pi \pi^{*}}$} & \multirow{2}{*}{ CASSCF $^{a}$} & \multicolumn{3}{|l|}{ RASSCF $^{D}$} & \multicolumn{3}{|c|}{ RASSCF $+3 p(0.5)^{c}$} & \multicolumn{3}{|c|}{$\operatorname{RASSCF}+3 p(1.0)^{c}$} & \multirow[b]{2}{*}{ CASPT2 } \\
\hline & & {$[1,1] \quad[1,2]$} & {$[2,1]$} & {$[2,2]$} & {$[1,1]$} & {$[1,2]$} & {$[2,1]$} & {$[1,1]$} & {$[1,2]$} & {$[2,1]$} & \\
\hline $\begin{array}{l}\pi \pi^{*} \\
1^{1} \mathrm{~B}_{2}{ }^{d}\end{array}$ & 5.03 & 5.49 & 5.49 & 5.45 & 5.44 & 5.32 & 5.43 & 5.47 & 5.32 & 5.46 & 5.02 \\
\hline $1^{1} \mathrm{~B}_{1}{ }^{\mathrm{d}}$ & 5.40 & 5.93 & 5.35 & 5.56 & 5.26 & 5.34 & 5.25 & 5.26 & 5.38 & 5.17 & 5.14 \\
\hline
\end{tabular}

${ }^{a}$ The active space is 8 electrons in 7 molecular orbitals $\left(12 n, 32 \pi, 32 \pi^{*}\right)$

${ }^{\mathrm{b}}$ The active space is 20 electrons in 19 molecular orbitals $(6,7,6)\left(62 \sigma, 12 n, 32 \pi, 32 \pi^{*}, 62 \sigma^{*}\right)$

${ }^{\mathrm{c}}$ The active space is 20 electrons in 25 molecular orbitals $(6,7,12)\left(62 \sigma, 12 n, 32 \pi, 32 \pi^{*}, 62 \sigma^{*}, 63 \pi^{*}\right)$

${ }^{\mathrm{d}}$ Excited state of covalent character (local $n \pi^{*}$ transition)

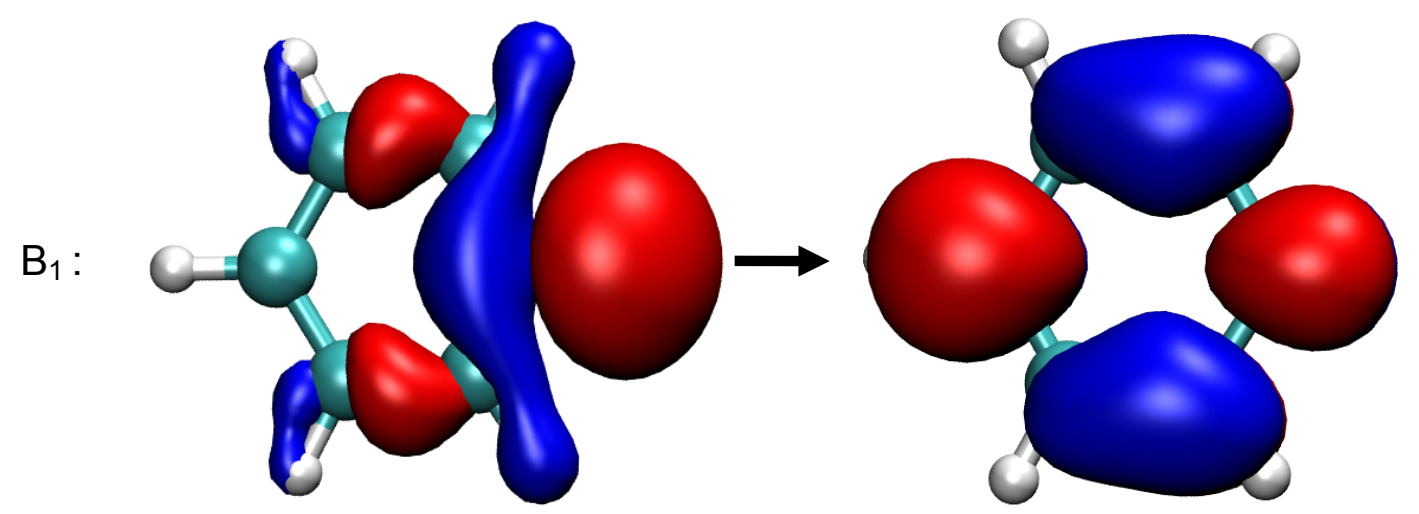

Figure S2: Molecular orbitals (isovalue of 0.03 ) involved in the two lowest $n \pi^{*}$ transition ( $\mathrm{B}_{1}$ character) of pyridine. 
Table S12: Vertical excitation energies $(\mathrm{eV})$ for the lowest singlet valence states of pyrazine obtained at the ground state MP2/6-31G* equilibrium geometry. The symmetry point group is $D_{2 h}$.

\begin{tabular}{|c|c|c|c|c|c|c|c|c|c|c|c|c|}
\hline \multirow[b]{2}{*}{ State } & \multirow[b]{2}{*}{ CASSCF $^{a}$} & \multicolumn{4}{|c|}{ RASSCF $^{D}$} & \multicolumn{3}{|c|}{ RASSCF $+3 p(0.5)^{C}$} & \multicolumn{3}{|c|}{ RASSCF+3p(1.0) } & \multirow[b]{2}{*}{ CASPT2 } \\
\hline & & {$[1,1]$} & {$[1,2]$} & {$[2,1]$} & {$[2,2]$} & {$[1,1]$} & {$[1,2]$} & {$[2,1]$} & {$[1,1]$} & {$[1,2]$} & {$[2,1]$} & \\
\hline$\pi \pi^{*}$ & & & & & & & & & & & & \\
\hline $\begin{array}{l}1^{1} \mathrm{~B}_{2 \mathrm{u}} \\
n \pi^{*}\end{array}$ & 4.94 & 5.34 & 5.31 & 5.35 & 5.31 & 5.27 & 5.16 & 5.31 & 5.28 & 5.16 & 5.32 & 4.85 \\
\hline $1^{1} B_{3 u}{ }^{d}$ & 4.78 & 4.70 & 4.99 & 4.56 & 4.79 & 4.48 & 4.50 & 4.40 & 4.49 & 4.51 & 4.41 & 4.12 \\
\hline $1^{1} A_{u}^{e}$ & 6.14 & 5.78 & 6.01 & 5.62 & 5.89 & 5.45 & 5.79 & 5.53 & 5.46 & 5.79 & 5.53 & 4.70 \\
\hline $1^{1} \mathrm{~B}_{2 \mathrm{~g}}$ & 5.85 & - & - & - & - & - & - & - & - & - & - & 5.68 \\
\hline
\end{tabular}

${ }^{a}$ The active space is 10 electrons in 8 molecular orbitals $\left(22 n, 32 \pi, 32 \pi^{*}\right)$. State-average over 5 states

${ }^{\mathrm{b}}$ The active space is 22 electrons in 20 molecular orbitals $(6,8,6)\left(62 \sigma, 22 n, 32 \pi, 32 \pi^{*}, 62 \sigma^{*}\right)$. State-average over 5 states

${ }^{\mathrm{c}}$ The active space is 22 electrons in 26 molecular orbitals $(6,8,12)\left(62 \sigma, 22 n, 32 \pi, 32 \pi^{*}, 62 \sigma^{*}, 63 \pi^{*}\right)$. Stateaverage over 5 states

${ }^{d}$ Excited state of covalent character (local $n \pi^{*}$ transition)

${ }^{\mathrm{e}}$ Excited state of ionic character (non-local $n \pi^{*}$ transition)

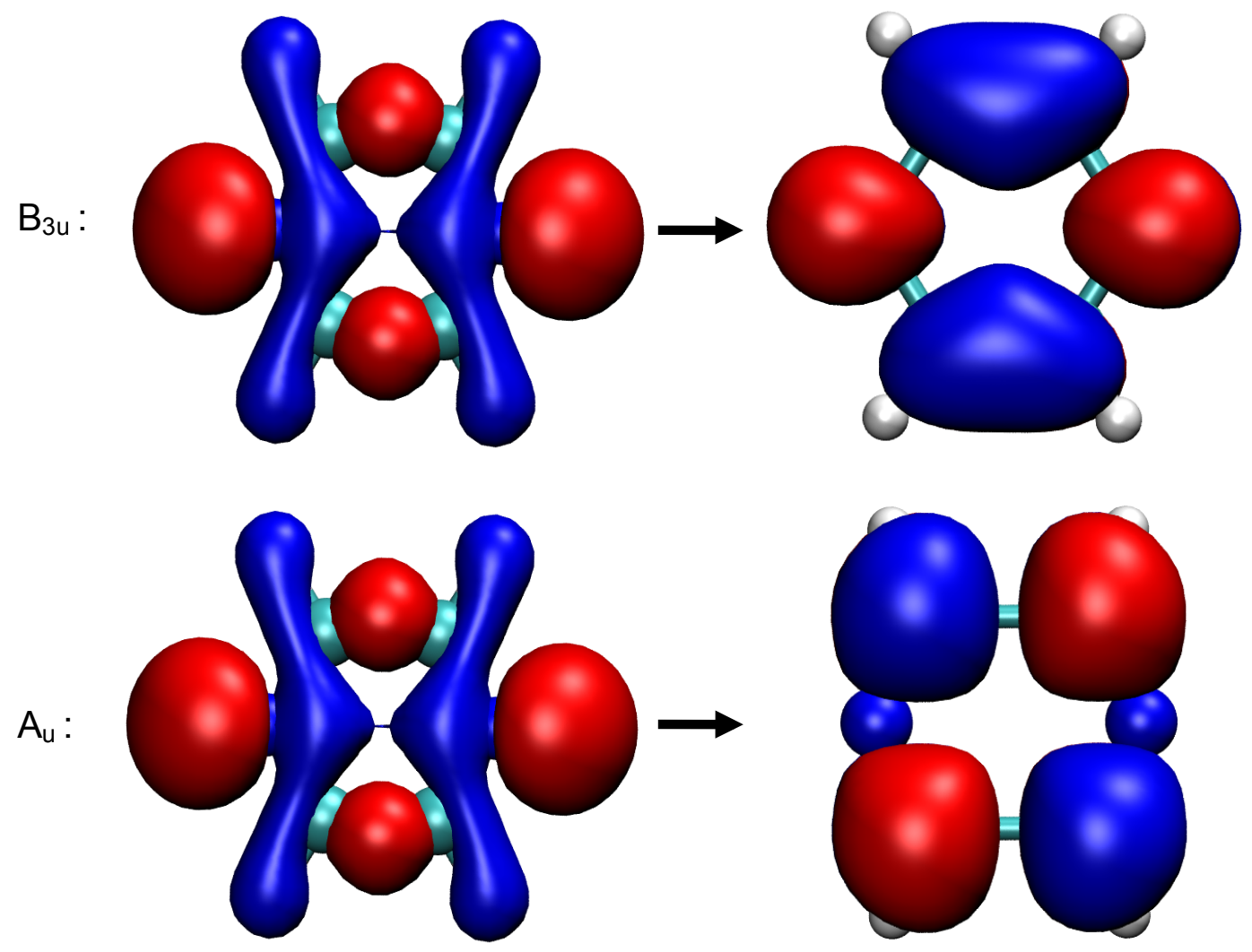

Figure S3: Molecular orbitals (isovalue of 0.03 ) involved in the two lowest $n \pi^{*}$ transition ( $B_{3 u}$ and $A_{u}$ character) of pyrazine. 
Table S13: Vertical excitation energies $(\mathrm{eV})$ for the lowest singlet valence states of pyrimidine obtained at the ground state MP2/6-31G* equilibrium geometry. The symmetry point group is $\mathrm{C}_{2 \mathrm{v}}$.

\begin{tabular}{|c|c|c|c|c|c|c|c|c|c|c|c|c|}
\hline \multirow{2}{*}{$\frac{\text { State }}{\pi \pi^{*}}$} & \multirow[b]{2}{*}{ CASSCF $^{a}$} & \multicolumn{4}{|c|}{ RASSCF $^{D}$} & \multicolumn{3}{|c|}{ RASSCF $+3 p(0.5)^{c}$} & \multicolumn{3}{|c|}{ RASSCF $+3 p(1.0)^{c}$} & \multirow[b]{2}{*}{ CASPT2 } \\
\hline & & {$[1,1]$} & {$[1,2]$} & {$[2,1]$} & {$[2,2]$} & {$[1,1]$} & {$[1,2]$} & {$[2,1]$} & {$[1,1]$} & {$[1,2]$} & {$[2,1]$} & \\
\hline $\begin{array}{l}\pi \pi^{*} \\
1^{1} \mathrm{~B}_{2} \\
n \pi^{*}\end{array}$ & 5.14 & 5.65 & 5.57 & 5.66 & 5.54 & - & - & - & - & - & - & 5.24 \\
\hline $\begin{array}{l}1^{1} \mathrm{~B}_{1}{ }^{\mathrm{d}} \\
1^{1} \mathrm{~A}_{2}\end{array}$ & $\begin{array}{l}4.92 \\
5.65\end{array}$ & $\begin{array}{l}4.89 \\
5.41\end{array}$ & $\begin{array}{l}5.34 \\
5.75\end{array}$ & $\begin{array}{l}4.81 \\
5.31\end{array}$ & $\begin{array}{l}5.24 \\
5.65\end{array}$ & $\begin{array}{l}4.65 \\
5.01\end{array}$ & $\begin{array}{l}4.86 \\
5.29\end{array}$ & $\begin{array}{l}4.68 \\
5.05\end{array}$ & $\begin{array}{l}4.65 \\
5.01\end{array}$ & $\begin{array}{l}4.87 \\
5.28\end{array}$ & $\begin{array}{l}4.68 \\
5.05\end{array}$ & $\begin{array}{l}4.44 \\
4.81\end{array}$ \\
\hline
\end{tabular}

${ }^{a}$ The active space is 10 electrons in 8 molecular orbitals $\left(22 n, 32 \pi, 32 \pi^{*}\right)$. State-average over 5 states

${ }^{\mathrm{b}}$ The active space is 22 electrons in 20 molecular orbitals $(6,8,6)\left(62 \sigma, 22 n, 32 \pi, 32 \pi^{*}, 62 \sigma^{*}\right)$. State-average over 4 states

${ }^{\mathrm{c}}$ The active space is 22 electrons in 26 molecular orbitals $(6,8,12)\left(62 \sigma, 22 n, 32 \pi, 32 \pi^{*}, 62 \sigma^{*}, 63 \pi^{*}\right)$

${ }^{\mathrm{d}}$ Excited state of covalent character (local $n \pi^{*}$ transition)

$\mathrm{B}_{1}$ :

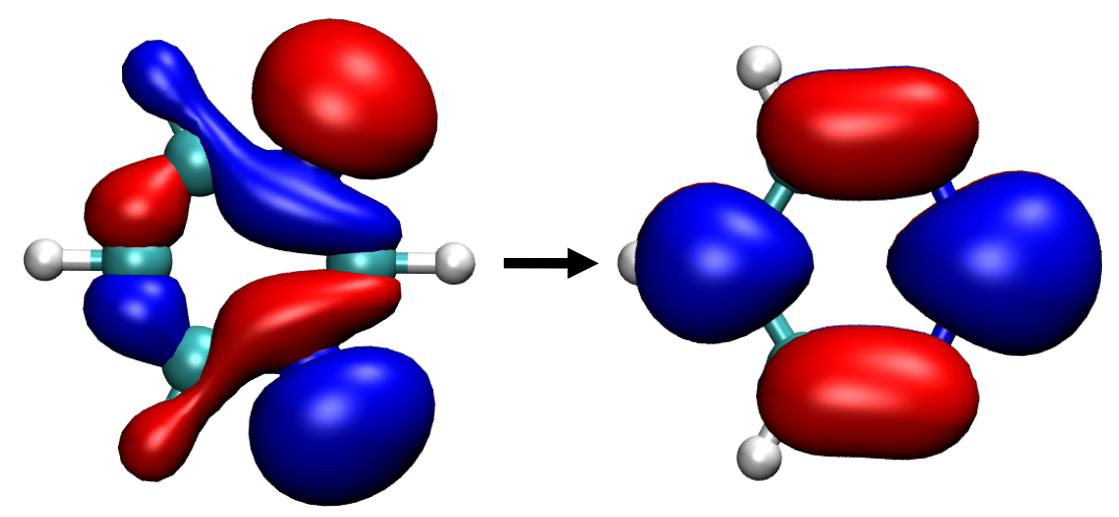

$\mathrm{A}_{2}$ :
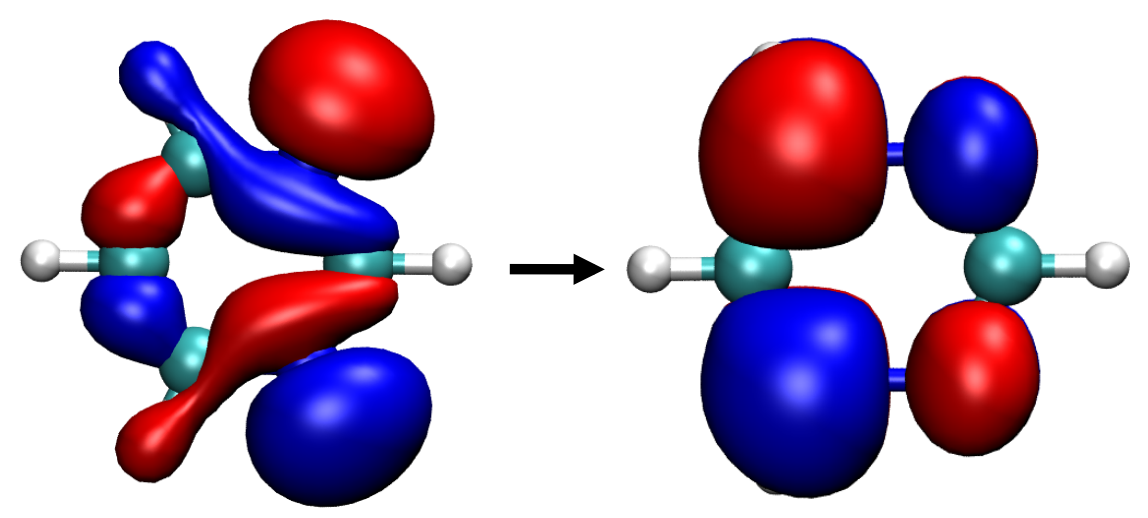

Figure S4: Molecular orbitals (isovalue of 0.03 ) involved in the two lowest $n \pi^{*}$ transition $\left(\mathrm{B}_{1}\right.$ and $\mathrm{A}_{2}$ character) of pyrimidine. 
Table S14: Vertical excitation energies $(\mathrm{eV})$ for the lowest singlet valence states of pyridazine obtained at the ground state MP2/6-31G* equilibrium geometry. The symmetry point group is $\mathrm{C}_{2 \mathrm{v}}$.

\begin{tabular}{|c|c|c|c|c|c|c|c|c|c|c|c|c|}
\hline \multirow[b]{2}{*}{ State } & \multirow[b]{2}{*}{ CASSCF $^{a}$} & \multicolumn{4}{|c|}{ RASSCF $^{D}$} & \multicolumn{3}{|c|}{ RASSCF+3p $(0.5)^{C}$} & \multicolumn{3}{|c|}{ RASSCF+3p(1.0) ${ }^{c}$} & \multirow[b]{2}{*}{ CASPT2 } \\
\hline & & {$[1,1]$} & {$[1,2]$} & {$[2,1]$} & {$[2,2]$} & {$[1,1]$} & {$[1,2]$} & {$[2,1]$} & {$[1,1]$} & {$[1,2]$} & {$[2,1]$} & \\
\hline$\pi \pi^{*}$ & & & & & & & & & & & & \\
\hline $\begin{array}{l}2^{1} \mathrm{~A}_{1} \\
n \pi^{*}\end{array}$ & 4.98 & - & - & - & - & - & - & - & - & - & - & 5.18 \\
\hline $1^{1} \mathrm{~B}_{1}{ }^{\mathrm{d}}$ & 4.37 & 4.28 & 4.75 & 4.17 & 4.41 & 4.02 & 4.28 & 3.98 & 4.03 & 4.28 & 4.02 & 3.78 \\
\hline $1^{1} \mathrm{~A}_{2}{ }^{\mathrm{d}}$ & 5.01 & 4.83 & 5.16 & 4.71 & 5.00 & 4.52 & 4.79 & 4.45 & 4.55 & 4.79 & 4.62 & 4.32 \\
\hline
\end{tabular}

${ }^{a}$ The active space is 10 electrons in 8 molecular orbitals $\left(22 n, 32 \pi, 32 \pi^{*}\right)$. State-average over 5 states

${ }^{\mathrm{b}}$ The active space is 22 electrons in 20 molecular orbitals $(6,8,6)\left(62 \sigma, 22 n, 32 \pi, 32 \pi^{*}, 62 \sigma^{*}\right)$

${ }^{\mathrm{c}}$ The active space is 22 electrons in 26 molecular orbitals $(6,8,12)\left(62 \sigma, 22 n, 32 \pi, 32 \pi^{*}, 62 \sigma^{*}, 63 \pi^{*}\right)$

${ }^{\mathrm{d}}$ Excited state of covalent character (local $n \pi^{*}$ transition)

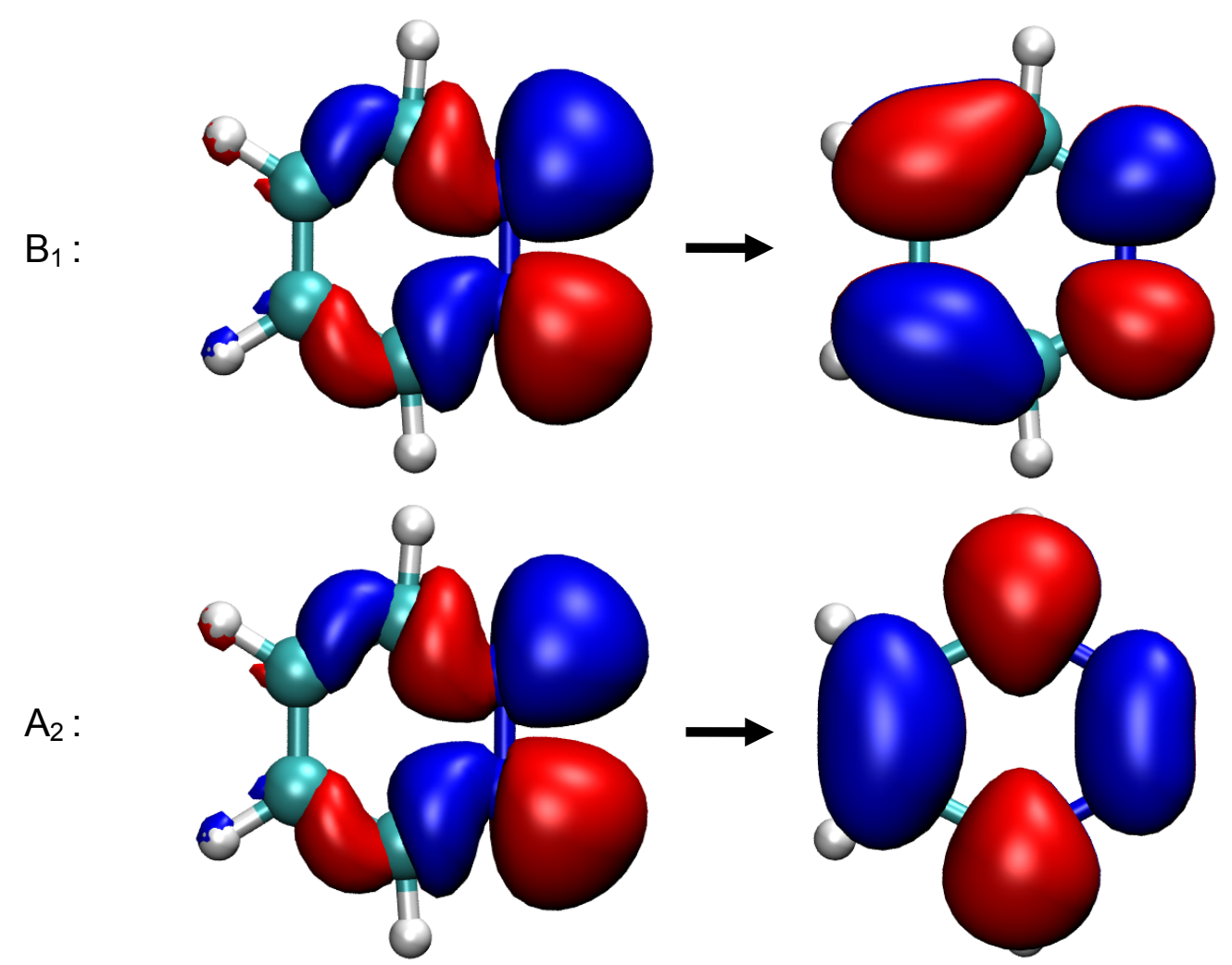

Figure S5: Molecular orbitals (isovalue of 0.03 ) involved in the two lowest $n \pi^{*}$ transition $\left(B_{1}\right.$ and $A_{2}$ character) of pyridazine. 
Table S19: Vertical excitation energies (eV) for the lowest singlet valence states of propanamide obtained at the ground state MP2/6-31G* equilibrium geometry. The symmetry point group is $\mathrm{C}_{\mathrm{s}}$.

\begin{tabular}{|c|c|c|c|c|c|c|c|c|c|c|c|c|}
\hline \multirow[b]{2}{*}{ State } & \multirow[b]{2}{*}{ CASSCF $^{a}$} & \multicolumn{4}{|c|}{ RASSCF $^{\text {D }}$} & \multicolumn{3}{|c|}{ RASSCF+3p $(0.5)^{C}$} & \multicolumn{3}{|c|}{ RASSCF+3p(1.0) } & \multirow[b]{2}{*}{ CASPT2 } \\
\hline & & {$[1,1]$} & {$[1,2]$} & {$[2,1]$} & {$[2,2]$} & {$[1,1]$} & {$[1,2]$} & {$[2,1]$} & {$[1,1]$} & {$[1,2]$} & {$[2,1]$} & \\
\hline $\begin{array}{l}\pi \pi^{*} \\
2^{1} A^{, d}\end{array}$ & 9.25 & 8.34 & 8.49 & 8.36 & 8.65 & 7.30 & 7.62 & 7.34 & 7.65 & 7.92 & 7.70 & 7.20 \\
\hline $1^{1} A^{\prime \prime e}$ & 5.66 & 5.89 & 6.31 & 5.86 & 6.00 & 5.65 & 5.89 & 5.64 & 5.72 & 5.87 & 5.73 & 5.72 \\
\hline
\end{tabular}

${ }^{a}$ The active space is 6 electrons in 4 molecular orbitals $\left(12 n, 22 \pi, 12 \pi^{*}\right)$

${ }^{\mathrm{b}}$ The active space is 14 electrons in 12 molecular orbitals $(4,4,4)\left(42 \sigma, 12 n, 22 \pi, 12 \pi^{*}, 42 \sigma^{*}\right)$

${ }^{\mathrm{c}}$ The active space is 14 electrons in 16 molecular orbitals $(4,4,8)\left(42 \sigma, 12 n, 22 \pi, 12 \pi^{*}, 42 \sigma^{*}, 33 \pi^{*}, 13 n^{*}\right)$

${ }^{d}$ Excited state of ionic character

e Excited state of covalent character 
Table S20: Vertical excitation energies $(\mathrm{eV})$ for the lowest singlet valence states of the investigated molecules computed with CASSCF, RASSCF and CASPT2.

\begin{tabular}{|c|c|c|c|c|c|c|}
\hline Molecules & States & CASSCF $^{\mathrm{a}}$ & RASSCF $^{\mathrm{b}}$ & MS-CASPT2 $^{\mathrm{d}}$ & MS-CASPT2 $^{\mathrm{e}}$ & SS-CASPT2 $^{\dagger}$ \\
\hline Ethene & $1^{1} B_{1 u}$ & 10.25 & $8.35^{\mathrm{c}}$ & 8.62 & 7.84 & 7.98 \\
\hline \multirow[t]{2}{*}{ E-butadiene } & $1^{1} \mathrm{Bu}$ & 8.63 & 6.82 & 6.47 & 6.38 & 6.23 \\
\hline & & 6.69 & 6.75 & 6.83 & 6.43 & 6.27 \\
\hline \multirow[t]{2}{*}{ E-hexatriene } & $1^{1} \mathrm{~B}_{\mathrm{u}}$ & 7.58 & 6.20 & 5.31 & 5.18 & 5.01 \\
\hline & $2^{1} A_{g}$ & 5.50 & 5.76 & 5.42 & 5.33 & 5.20 \\
\hline \multirow[t]{2}{*}{ E-octatetraene } & $2^{1} A_{g}$ & 4.74 & 4.86 & 4.64 & 4.52 & 4.38 \\
\hline & $1^{1} \mathrm{~B}_{\mathrm{u}}$ & 5.92 & 5.60 & 4.70 & 4.35 & 4.42 \\
\hline Cyclopropene & $1^{1} \mathrm{~B}_{2}$ & 9.20 & 7.16 & 7.06 & 6.66 & 7.45 \\
\hline \multirow[t]{2}{*}{ Cyclopentadiene } & $1^{1} \mathrm{~B}_{2}$ & 7.74 & 6.13 & 5.51 & 5.43 & 5.27 \\
\hline & $2^{1} \mathrm{~A}_{1}$ & 6.61 & 6.67 & 6.31 & 6.28 & 6.31 \\
\hline \multirow[t]{2}{*}{ Benzene } & $1^{1} \mathrm{~B}_{2 \mathrm{u}}$ & 4.89 & $5.18^{c}$ & 5.05 & 4.96 & 4.84 \\
\hline & $1^{1} \mathrm{~B}_{1 \mathrm{u}}$ & 8.27 & $7.19^{c}$ & 6.45 & 6.57 & 6.30 \\
\hline \multirow[t]{2}{*}{ Furan } & $1^{1} \mathrm{~B}_{2}$ & 8.36 & 6.51 & 6.43 & 6.19 & 6.04 \\
\hline & $2^{1} \mathrm{~A}_{1}$ & 6.81 & 6.77 & 6.52 & 6.35 & 6.16 \\
\hline \multirow[t]{2}{*}{ Pyrrole } & $2^{1} \mathrm{~A}_{1}$ & 6.63 & $6.61^{c}$ & 6.31 & 6.23 & 5.92 \\
\hline & $1^{1} \mathrm{~B}_{2}$ & 8.17 & $6.96^{c}$ & 6.33 & 6.22 & 6.00 \\
\hline \multirow[t]{2}{*}{ Imidazole } & $2^{1} A^{\prime}$ & 6.92 & 6.63 & 6.19 & 6.40 & 6.72 \\
\hline & $1^{1} \mathrm{~A} "$ & 7.18 & 6.91 & 6.81 & 6.69 & 6.52 \\
\hline \multirow[t]{2}{*}{ Pyridine } & $1^{1} \mathrm{~B}_{2}$ & 5.03 & 5.44 & 5.02 & 5.00 & 4.84 \\
\hline & $1^{1} \mathrm{~B}_{1}$ & 5.40 & 5.26 & 5.14 & 5.17 & 4.91 \\
\hline \multirow[t]{2}{*}{ Pyrazine } & $1^{1} B_{3 u}$ & 4.78 & 4.48 & 4.12 & 4.02 & 3.63 \\
\hline & $1^{1} A_{u}$ & 6.15 & 5.45 & 4.70 & 4.75 & 4.52 \\
\hline \multirow[t]{2}{*}{ Pyrimidine } & $1^{1} \mathrm{~B}_{1}$ & 4.92 & 4.65 & 4.44 & 4.34 & 3.81 \\
\hline & $1^{1} \mathrm{~A}_{2}$ & 5.66 & 5.01 & 4.81 & 4.74 & 4.12 \\
\hline \multirow[t]{2}{*}{ Pyridazine } & $1^{1} \mathrm{~B}_{1}$ & 4.37 & 4.02 & 3.78 & 3.71 & 3.48 \\
\hline & $1^{1} A_{2}$ & 5.01 & 4.52 & 4.32 & 4.18 & 3.66 \\
\hline \multirow[t]{2}{*}{ Formaldehyde } & $1^{1} A_{2}$ & 4.12 & 4.32 & 3.98 & 4.01 & 3.91 \\
\hline & $2^{1} \mathrm{~A}_{1}$ & 11.90 & 9.93 & 9.31 & 9.47 & 9.77 \\
\hline \multirow[t]{2}{*}{ Acetone } & $1^{1} \mathrm{~A}_{2}$ & 4.44 & 4.43 & 4.42 & 4.49 & 4.18 \\
\hline & $2^{1} A_{1}$ & 11.31 & 9.92 & 9.31 & 9.19 & 9.16 \\
\hline \multirow[t]{2}{*}{ Formamide } & $1^{1} A^{\prime \prime}$ & 5.60 & 5.66 & 5.63 & 5.58 & 5.61 \\
\hline & $2^{1} A^{\prime}$ & 9.33 & 7.63 & 7.44 & 7.45 & 7.41 \\
\hline \multirow[t]{2}{*}{ Acetamide } & $1^{1} A "$ & 5.62 & 5.63 & 5.80 & 5.69 & 5.54 \\
\hline & $2^{1} A^{\prime}$ & 9.26 & 7.33 & 7.27 & 7.12 & 7.21 \\
\hline \multirow[t]{2}{*}{ Propanamide } & $1^{1} A^{\prime \prime}$ & 5.66 & 5.65 & 5.72 & 5.74 & 5.48 \\
\hline & $2^{1} A^{\prime}$ & 9.25 & 7.30 & 7.20 & 7.17 & 7.28 \\
\hline \multicolumn{7}{|c|}{$\begin{array}{l}{ }^{a} \text { Result with } 6-31 G^{*} \text { basis set from this work } \\
{ }^{b} \text { Result from RASSCF }[1,1]+3 p(0.5) \text { from this work } \\
{ }^{c} \text { Result using scaling factor of } 1.0 \text { for extra basis basis } \\
{ }^{d} \text { Result with TZVP basis set from Ref. } 1 \\
{ }^{e} \text { Result with Aug-CC-pVTZ basis set from Ref. } 2 \\
{ }^{f} \text { Result with ANO basis set from Ref. } 1\end{array}$} \\
\hline
\end{tabular}


Table S21: Vertical excitation energies $(\mathrm{eV})$ for the lowest singlet valence states of the investigated molecules computed with Algebraic diagrammatic construction (ADC) and coupled-cluster method (CC).

\begin{tabular}{|c|c|c|c|c|c|c|c|c|}
\hline Molecules & States & $\operatorname{ADC}(2)-s^{a}$ & $\operatorname{ADC}(2)-x^{a}$ & $\operatorname{ADC}(3)^{a}$ & $\mathrm{CC} 2^{\mathrm{b}}$ & $\mathrm{CC}^{\mathrm{b}}$ & $\mathrm{CC}^{\mathrm{C}}$ & $\mathrm{CC}^{\mathrm{C}}$ \\
\hline Ethene & $1^{1} \mathrm{~B}_{1 \mathrm{u}}$ & 8.36 & 8.00 & 8.14 & 8.40 & 8.37 & 7.90 & 7.89 \\
\hline \multirow[t]{2}{*}{ E-butadiene } & $1^{1} \mathrm{Bu}$ & 6.43 & 5.93 & 6.36 & 6.49 & 6.58 & & \\
\hline & $2^{1} A_{g}$ & 7.68 & 5.12 & 5.77 & 7.63 & 6.77 & 7.06 & 6.63 \\
\hline \multirow[t]{2}{*}{ E-hexatriene } & $1^{1} B_{u}^{9}$ & 5.35 & 4.85 & 5.35 & 5.41 & 5.58 & & \\
\hline & $2^{1} A_{g}$ & 6.72 & 4.02 & 4.52 & 6.67 & 5.72 & & \\
\hline \multirow[t]{2}{*}{ E-octatetraene } & $2^{1} A_{g}$ & 5.93 & 3.30 & 3.73 & 5.87 & 4.97 & & \\
\hline & $1^{1} \mathrm{~B}_{\mathrm{u}}$ & 4.66 & 4.17 & 4.70 & 4.72 & 4.94 & & \\
\hline Cyclopropene & $1^{1} \mathrm{~B}_{2}$ & 7.14 & 6.60 & 6.91 & 7.17 & 7.10 & 6.72 & 6.68 \\
\hline \multirow[t]{2}{*}{ Cyclopentadiene } & $1^{1} \mathrm{~B}_{2}$ & 5.66 & 5.15 & 5.52 & 5.59 & 5.73 & 5.47 & \\
\hline & $2^{1} A_{1}$ & 7.08 & 5.11 & 5.81 & 7.05 & 6.61 & 6.80 & \\
\hline \multirow[t]{2}{*}{ Benzene } & $1^{1} \mathrm{~B}_{2 \mathrm{u}}$ & 5.27 & 4.22 & 4.99 & 5.27 & 5.07 & 5.22 & 5.03 \\
\hline & $1^{1} \mathrm{~B}_{1 \mathrm{u}}$ & 6.64 & 6.20 & 6.47 & 6.68 & 6.68 & 6.45 & 6.42 \\
\hline \multirow[t]{2}{*}{ Furan } & $1^{1} \mathrm{~B}_{2}$ & 6.76 & 6.16 & 6.39 & 6.75 & 6.60 & 6.37 & \\
\hline & $2^{1} \mathrm{~A}_{1}$ & 6.85 & 5.52 & 6.48 & 6.87 & 6.62 & 6.70 & \\
\hline \multirow[t]{2}{*}{ Pyrrole } & $2^{1} \mathrm{~A}_{1}$ & 6.60 & 5.46 & 6.38 & 6.61 & 6.40 & 6.42 & \\
\hline & $1^{1} \mathrm{~B}_{2}$ & 6.89 & 6.20 & 6.53 & 6.83 & 6.71 & 6.85 & \\
\hline \multirow[t]{2}{*}{ Imidazole } & $2^{1} A^{\prime}$ & 6.73 & 5.67 & 6.49 & 6.73 & 6.58 & 6.40 & \\
\hline & $1^{1} A^{\prime \prime}$ & 6.74 & 6.05 & 6.46 & 6.86 & 6.82 & 6.69 & \\
\hline \multirow[t]{2}{*}{ Pyridine } & $1^{1} \mathrm{~B}_{2}$ & 5.32 & 4.28 & 5.06 & 5.32 & 5.15 & 5.26 & \\
\hline & $1^{1} \mathrm{~B}_{1}$ & 5.10 & 4.21 & 5.05 & 5.12 & 5.05 & 4.97 & \\
\hline \multirow[t]{2}{*}{ Pyrazine } & $1^{1} \mathrm{~B}_{3 \mathrm{u}}$ & 4.29 & 3.45 & 4.21 & 4.26 & 4.24 & 4.12 & \\
\hline & $1^{1} A_{u}$ & 4.97 & 4.06 & 5.28 & 4.95 & 5.05 & 4.86 & \\
\hline \multirow[t]{2}{*}{ Pyrimidine } & $1^{1} \mathrm{~B}_{1}$ & 4.45 & 3.59 & 4.57 & 4.49 & 4.50 & 4.37 & \\
\hline & $1^{1} \mathrm{~A}_{2}$ & 4.80 & 3.92 & 5.10 & 4.84 & 4.93 & 4.75 & \\
\hline \multirow[t]{2}{*}{ Pyridazine } & $1^{1} \mathrm{~B}_{1}$ & 3.91 & 3.02 & 3.92 & 3.90 & 3.92 & 3.79 & \\
\hline & $1^{1} \mathrm{~A}_{2}$ & 4.41 & 3.40 & 4.68 & 4.40 & 4.49 & 4.30 & \\
\hline \multirow[t]{2}{*}{ Formaldehyde } & $1^{1} A_{2}$ & 3.91 & 3.10 & 3.85 & 4.09 & 3.95 & 4.40 & \\
\hline & $2^{1} \mathrm{~A}_{1}$ & 9.37 & 8.71 & 9.09 & 10.34 & 10.45 & & \\
\hline \multirow[t]{2}{*}{ Acetone } & $1^{1} A_{2}$ & 4.30 & 3.54 & 4.38 & 4.52 & 4.40 & 4.45 & \\
\hline & $2^{1} A_{1}$ & 9.44 & 8.70 & 9.96 & 9.74 & 9.65 & 9.31 & \\
\hline \multirow[t]{2}{*}{ Formamide } & $1^{1} A^{\prime \prime}$ & 5.46 & 4.77 & 5.69 & 5.76 & 5.65 & 5.59 & 5.55 \\
\hline & $2^{1} A^{\prime}$ & 7.82 & 7.26 & 7.48 & 8.15 & 8.27 & & \\
\hline \multirow[t]{2}{*}{ Acetamide } & $1^{1} A^{\prime \prime}$ & 5.48 & 4.80 & 5.78 & 5.77 & 5.69 & 5.61 & \\
\hline & $2^{1} A^{\prime}$ & 7.47 & 6.81 & 7.59 & 7.66 & 7.67 & 7.23 & \\
\hline \multirow[t]{2}{*}{ Propanamide } & $1^{1} A^{\prime \prime}$ & 5.49 & 4.82 & 5.81 & 5.78 & 5.72 & 5.62 & \\
\hline & $2^{1} A^{\prime}$ & 7.39 & 6.75 & 7.58 & 7.56 & 7.62 & 7.16 & \\
\hline
\end{tabular}

\footnotetext{
${ }^{a}$ Result with TZVP basis set from ref. 3

${ }^{\mathrm{b}}$ Result with TZVP basis set from ref. 1

${ }^{c}$ Result with Aug-cc-pVTZ basis set from ref. 4
}

Table S22: Vertical excitation energies $(\mathrm{eV})$ for the lowest singlet valence states of the investigated molecules computed with time-dependent density functional theory, Bethe-Salpeter and SOPPA method. Theoretical best estimate (TBE) provided for comparison for all methods.

\begin{tabular}{lcccccc}
\multicolumn{1}{l}{} \\
\hline Molecules & States & TD-B3LYP & BSE/evGW@PBE0 & SOPPA $^{\mathrm{C}}$ & TBE-1 $^{\mathrm{d}}$ & TBE-2 $^{\mathrm{e}}$ \\
\hline Ethene & $1^{1} \mathrm{~B}_{1 \mathrm{u}}$ & 7.70 & 7.32 & 7.84 & 7.80 & 7.80 \\
E-butadiene & $1^{1} \mathrm{Bu}$ & 5.74 & 5.71 & 5.88 & 6.18 & 6.18 \\
\hline
\end{tabular}




\begin{tabular}{|c|c|c|c|c|c|c|}
\hline & $2 \mathrm{~A}_{\mathrm{g}}$ & 6.82 & 6.58 & 7.29 & 6.55 & 6.55 \\
\hline \multirow[t]{2}{*}{ E-hexatriene } & $1^{1} B_{u}$ & 4.69 & 4.79 & 4.80 & 5.10 & 5.10 \\
\hline & $2^{1} A_{g}$ & 5.69 & 6.11 & 6.30 & 5.09 & 5.09 \\
\hline \multirow[t]{2}{*}{ E-octatetraene } & $2^{1} A_{g}$ & 4.84 & 5.52 & 5.49 & 4.47 & 4.47 \\
\hline & $1^{1} B_{u}$ & 4.02 & 4.15 & 4.12 & 4.66 & 4.66 \\
\hline Cyclopropene & $1^{1} \mathrm{~B}_{2}$ & 6.31 & 6.14 & 6.65 & 7.06 & 6.68 \\
\hline \multirow[t]{2}{*}{ Cyclopentadiene } & $1^{1} \mathrm{~B}_{2}$ & 5.02 & 4.88 & 5.11 & 5.55 & 5.55 \\
\hline & $2^{1} \mathrm{~A}_{1}$ & 6.52 & 6.50 & 6.63 & 6.31 & 6.28 \\
\hline \multirow[t]{2}{*}{ Benzene } & $1^{1} \mathrm{~B}_{2 \mathrm{u}}$ & 5.40 & 5.04 & 4.69 & 5.08 & 5.08 \\
\hline & $1^{1} \mathrm{~B}_{1 \mathrm{u}}$ & 6.10 & 5.84 & 6.15 & 6.54 & 6.54 \\
\hline \multirow[t]{2}{*}{ Furan } & $1^{1} \mathrm{~B}_{2}$ & 6.16 & 5.78 & 6.23 & 6.32 & 6.32 \\
\hline & $2^{1} \mathrm{~A}_{1}$ & 6.70 & 6.38 & 6.33 & 6.57 & 6.57 \\
\hline \multirow[t]{2}{*}{ Pyrrole } & $2^{1} A_{1}$ & 6.53 & 6.01 & 6.08 & 6.37 & 6.37 \\
\hline & $1^{1} B_{2}$ & 6.40 & 6.67 & 6.38 & 6.57 & 6.57 \\
\hline \multirow[t]{2}{*}{ Imidazole } & $2^{1} A^{\prime}$ & 6.45 & 5.96 & 6.90 & 6.19 & 6.25 \\
\hline & $1^{1} A^{\prime \prime}$ & 6.46 & 6.24 & 6.32 & 6.81 & 6.65 \\
\hline \multirow[t]{2}{*}{ Pyridine } & $1^{1} \mathrm{~B}_{2}$ & 5.49 & 5.08 & 4.70 & 4.85 & 4.85 \\
\hline & $1^{1} \mathrm{~B}_{1}$ & 4.80 & 4.79 & 4.58 & 4.59 & 4.59 \\
\hline \multirow[t]{2}{*}{ Pyrazine } & $1^{1} \mathrm{~B}_{3 \mathrm{u}}$ & 3.96 & 3.90 & 3.72 & 3.95 & 4.13 \\
\hline & $1^{1} \mathrm{~A}_{\mathrm{u}}$ & 4.69 & 4.81 & 4.50 & 4.81 & 4.98 \\
\hline \multirow[t]{2}{*}{ Pyrimidine } & $1^{1} \mathrm{~B}_{1}$ & 4.27 & 4.26 & 3.93 & 4.55 & 4.43 \\
\hline & $1^{1} \mathrm{~A}_{2}$ & 4.60 & 4.17 & 4.32 & 4.91 & 4.85 \\
\hline \multirow[t]{2}{*}{ Pyridazine } & $1^{1} \mathrm{~B}_{1}$ & 3.58 & 3.57 & 3.31 & 3.78 & 3.85 \\
\hline & $1^{1} \mathrm{~A}_{2}$ & 4.18 & 4.28 & 3.91 & 4.32 & 4.44 \\
\hline \multirow[t]{2}{*}{ Formaldehyde } & $1^{1} \mathrm{~A}_{2}$ & 3.89 & 3.61 & 3.45 & 3.88 & 3.88 \\
\hline & $2^{1} A_{1}$ & 9.17 & 8.95 & 9.55 & 9.30 & 9.29 \\
\hline \multirow[t]{2}{*}{ Acetone } & $1^{1} \mathrm{~A}_{2}$ & 4.34 & 3.97 & 3.82 & 4.40 & 4.38 \\
\hline & $2^{1} A_{1}$ & 9.04 & 8.67 & 8.96 & 9.40 & 8.90 \\
\hline \multirow[t]{2}{*}{ Formamide } & $1^{1} A^{\prime \prime}$ & 5.55 & 5.59 & 5.00 & 5.63 & 5.55 \\
\hline & $2^{1} A^{\prime}$ & 8.13 & 7.48 & 7.48 & 7.44 & 7.35 \\
\hline \multirow[t]{2}{*}{ Acetamide } & $1^{1} A^{\prime \prime}$ & 5.56 & 5.56 & 5.01 & 5.80 & 5.62 \\
\hline & $2^{1} A^{\prime}$ & 7.46 & 7.56 & 7.02 & 7.27 & 7.14 \\
\hline \multirow[t]{2}{*}{ Propanamide } & $1^{1} A^{\prime \prime}$ & 5.59 & 5.55 & 5.02 & 5.72 & 5.65 \\
\hline & $2^{1} A^{\prime}$ & 7.76 & 7.53 & 6.96 & 7.20 & 7.09 \\
\hline
\end{tabular}

\footnotetext{
${ }^{a}$ Result with TZVP basis set from ref. 5

${ }^{\mathrm{b}}$ Result with Aug-cc-pVTZ basis set from ref. 6

${ }^{c}$ Result with TZVP basis set from ref. 7

${ }^{d}$ Theoretical best estimate taken from ref. 1

e Theoretical best estimate taken from ref. 2
} 


\section{Error comparison}

\section{CASSCF}

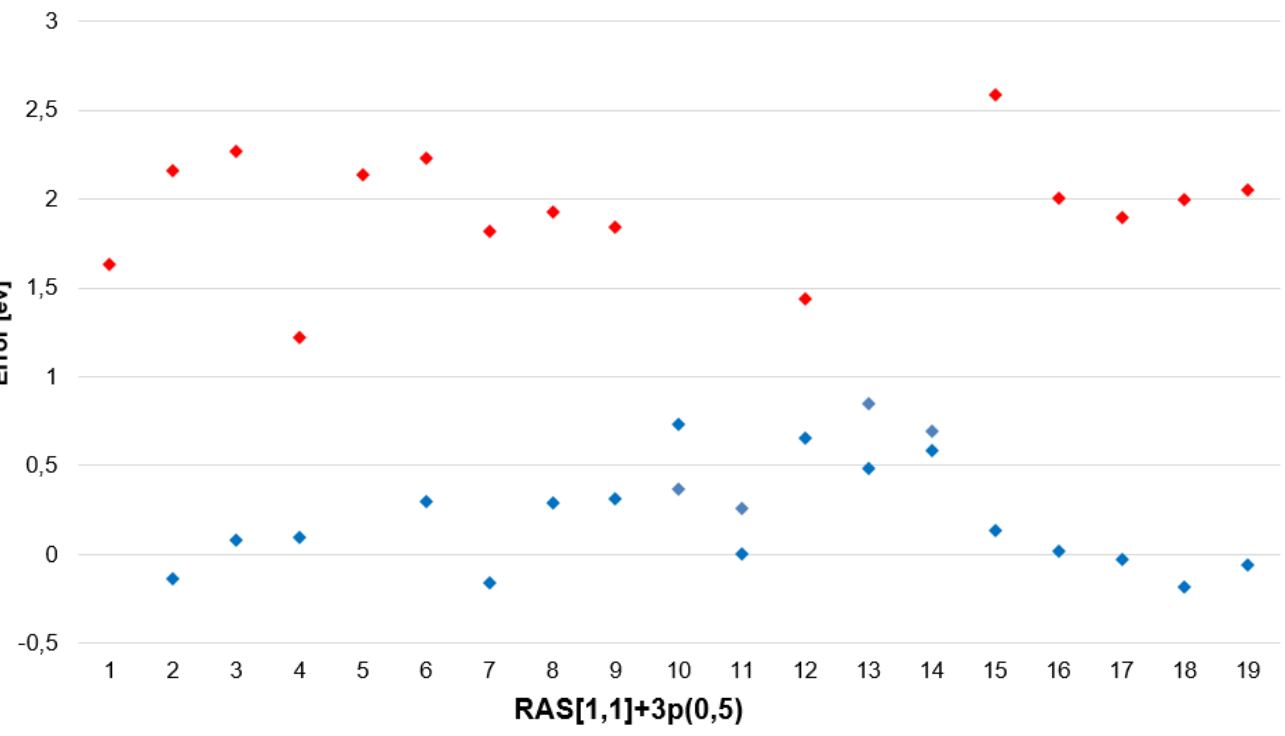

3

2,5

2

$\underbrace{\overbrace{}^{3}}{ }^{1,5}$

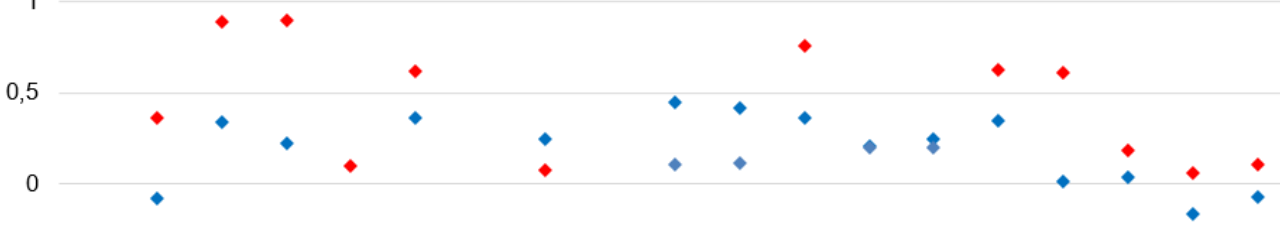

$-0,5$

$\begin{array}{lllllllllllllllllll}1 & 2 & 3 & 4 & 5 & 6 & 7 & 8 & 9 & 10 & 11 & 12 & 13 & 14 & 15 & 16 & 17 & 18 & 19\end{array}$ $\operatorname{RAS}[1,1]+3 p(1,0)$

2,5

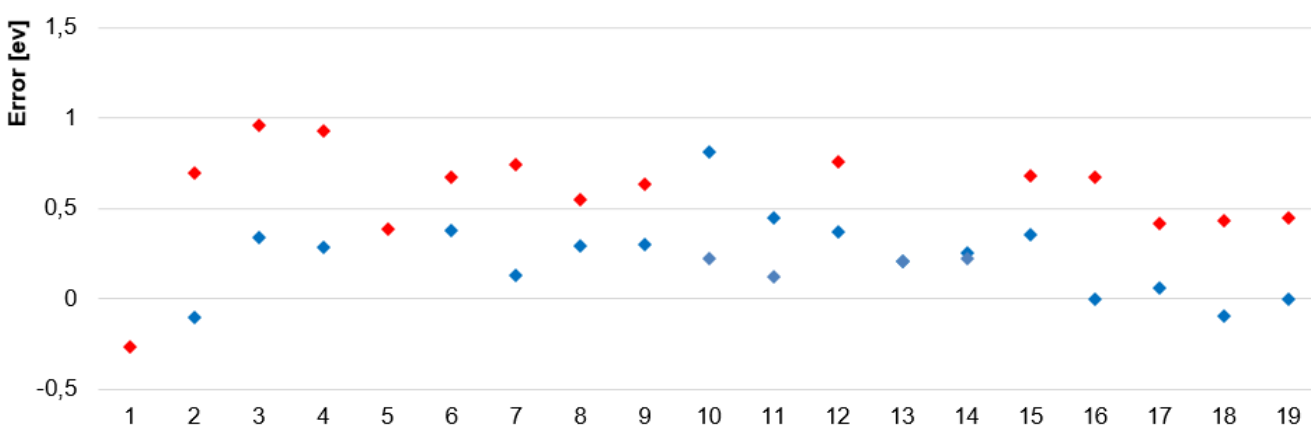

Figure S6: Error comparison between the 19 molecules with covalent states in blue and ionic state in red from computation at CASSCF, RAS $[1,1]+3 p(0.5)$ and RAS $[1,1]+3 p(1.0)$. The Rydberg state are not included (missing data points in RAS $[1,1]+3 p(0.5))$. The errors are relative to MS-CASPT2/TZVP results. 


\section{References}

(1) Schreiber, M.; Silva-Junior, M. R.; Sauer, S. P. A.; Thiel, W. Benchmarks for Electronically Excited States: CASPT2, CC2, CCSD, and CC3. J. Chem. Phys. 2008, 128, 134110.

(2) Silva-Junior, M. R.; Schreiber, M.; Sauer, S. P. A.; Thiel, W. Benchmarks of Electronically Excited States: Basis Set Effects on CASPT2 Results. J. Chem. Phys. 2010, 133 , 174318.

(3) Harbach, P. H. P.; Wormit, M.; Dreuw, A. The Third-Order Algebraic Diagrammatic Construction Method (ADC(3)) for the Polarization Propagator for Closed-Shell Molecules: Efficient Implementation and Benchmarking. J. Chem. Phys. 2014, 141, 064113.

(4) Silva-Junior, M. R.; Sauer, S. P. A.; Schreiber, M.; Thiel, W. Basis Set Effects on Coupled Cluster Benchmarks of Electronically Excited States: CC3, CCSDR(3) and CC2. Mol. Phys. 2010, $108,453-465$.

(5) Silva-Junior, M. R.; Schreiber, M.; Sauer, S. P. A.; Thiel, W. Benchmarks for Electronically Excited States: Time-Dependent Density Functional Theory and Density Functional Theory Based Multireference Configuration Interaction. J. Chem. Phys. 2008, 129, 104103.

(6) Jacquemin, D.; Duchemin, I.; Blase, X. Benchmarking the Bethe-Salpeter Formalism on a Standard Organic Molecular Set. J. Chem. Theory Comput. 2015, 11, 3290-3304.

(7) Sauer, S. P. A.; Pitzner-Frydendahl, H. F.; Buse, M.; Jensen, H. J. A.; Thiel, W. Performance of SOPPA-Based Methods in the Calculation of Vertical Excitation Energies and Oscillator Strengths. Mol. Phys. 2015, 113, 2026-2045. 